\title{
Metagenomic analysis of viral communities in diseased Baikal sponge Lubomirskia baikalensis
}

\author{
Butina T.V. ${ }^{1, *}$, Bukin Yu.S. ${ }^{1,2}$, Khanaev I.V. ${ }^{1}$, Kravtsova L.S. ${ }^{1}$, Maikova O.O. ${ }^{1}$, \\ Tupikin A.E. ${ }^{3}$, Kabilov M.R. ${ }^{3}$, Belikov S.I. ${ }^{1}$
}

\begin{abstract}
${ }^{1}$ Limnological Institute, Siberian Branch of the Russian Academy of Sciences, Ulan-Batorskaya Str., 3, Irkutsk, 664033, Russia ${ }^{2}$ Irkutsk National Research Technical University, Lermontov Str., 83, Irkutsk, 664074, Russia

${ }^{3}$ Institute of Chemical Biology and Fundamental Medicine, Siberian Branch of the Russian Academy of Sciences, Lavrentiev Ave., 8, Novosibirsk, 630090, Russia
\end{abstract}

\begin{abstract}
Sponges are an ecologically important component of marine and freshwater bodies. Sponge community includes a variety of microorganisms: fungi, algae, archaea, bacteria and viruses. Despite active research in the field of aquatic virology, biodiversity and the role of viruses in sponges are poorly studied. The relevance of research in this area is also related to the worldwide problem of sponge diseases. The aim of this study was to elucidate the genetic diversity of viruses in the associated community of diseased endemic Baikal sponge Lubomirskia baikalensis using metagenomic analysis. As a result, we have shown for the first time a high genetic and taxonomic diversity of DNA viruses in the Baikal sponge community. Identified sequences belonged to 16 viral families that infect a wide range of organisms. Moreover, our analysis indicated the differences in viral communities of visually healthy and diseased branches of the sponge. The approach used in this study is promising for further studies of viral communities in sponges, obtaining more complete information about the taxonomic and functional diversity of viruses in holobionts and entire Lake Baikal, and identifying the role of viruses in sponge diseases.
\end{abstract}

Keywords: metagenomic analysis, virome, viral diversity, viral communities, sponges, Lake Baikal

\section{Introduction}

Viruses are the most ubiquitous, abundant and diverse biological objects on Earth. They are of particular importance in the water environments, where their concentration reaches $10^{11}$ particles $/ \mathrm{ml}$ (Wilhelm and Matteson, 2008). They regulate the abundance, composition and biodiversity of numerous aquatic microorganisms and other hydrobionts, participate in biochemical processes and, in general, influence significantly on the functioning and ecological state of water bodies (Wommack and Colwell, 2000; Suttle, 2007; Jacquet et al., 2015). Despite the considerable advances in the field of aquatic virology in recent decades, we lack knowledge about viruses of marine and, especially, freshwater invertebrates including symbiotic organisms, or it is incomplete.

Sponges are the oldest multicellular invertebrates (phylum Porifera) that represent complex symbioses in marine and freshwater ecosystems and have unusual properties: high diversity, abundance and biomass; contribution to primary production and nitrification though symbioses; high chemical and physical adaptation; competitiveness; biomineralization; production of the secondary metabolites, etc. (Diaz and Ruetzler, 2001; Bell, 2008). The sponge community includes various microorganisms: fungi, dinoflagellates, small algae, archaea, bacteria, and viruses. The abundance and diversity of viruses can be very significant, considering a great number of their potential hosts in the sponge community. However, the diversity and importance of viruses have been little studied compared to other spongeassociated microorganisms, and the role of viruses in the sponges remains largely unknown. Previously, virus-like particles (VLPs) morphologically similar to adenoviruses, picornaviruses and mimiviruses were rarely found in some sponges (Vacelet and Gallissian, 1978; Johnson, 1984; Claverie et al., 2009). Recently, the first comprehensive morphological assessment of sponge-associated viruses by transmission electron microscopy has revealed diverse communities of virallike particles in the different marine sponge species (Pascelli et al, 2018). Moreover, the first metaviromic studies provided new molecular data on the composition, function of viruses inhabiting reef sponges, and showed a high diversity of sponge viral communities (Laffy et al., 2016; 2018). 
As natural filter feeders, sponges play a great role in the existence of any water body, which is especially relevant for Lake Baikal as a unique natural object characterized by a huge freshwater supply, ancient origin, a rich diversity of endemic flora and fauna, etc. (Kozhova and Izmesteva, 1998). According to the current taxonomy, two families represent the Baikal sponges: endemic Lubomirskiidae (includes 4 genera and 14 species) and cosmopolitan Spongillidae (3 genera and 5 species) (Efremova, 2001; 2004; Itskovich et al., 2017). Endemic species inhabit almost all depths of the lake (from $1 \mathrm{~m}$ to maximum depths), but the bulk of their abundance and species diversity is concentrated at depths of 5-30 m (Khanaev et al., 2018), where their biomass mostly exceeds the biomass of all zoobenthic groups (Kozhov, 1970). The most mass species Lubomirskia baikalensis is the only branching sponge in Lake Baikal, which colonies reach up to 1.5 $\mathrm{m}$ in height.

The first investigations of viruses associated with the Baikal sponges Lubomirskia baikalensis were based on the analysis of marker genes. We identified a high diversity of $g 20$ gene of cyanophages (Butina et al., 2015) and g23 genes of T4-like bacteriophages (Butina T.V., unpublished data) in the community of L. baikalensis. The analysis revealed specific groups of cyanophages different from those inhabiting the lake water, which is natural and corresponds to the hologenome concept. Holobiont is a united complex of the host organism (sponge, coral, etc.) and associated microorganisms (Rohwer et al., 2002). The genetic material of holobiont (hologenome) changes faster than the genome of the host organism, which increases the potential and adaptability of the complex organism in unfavourable and changing conditions (Rosenberg et al., 2007). Sponges are one of the most diverse and complex aquatic holobionts (Webster and Thomas, 2016; Pita et al., 2018).

Over the past decades, Lake Baikal has experienced an increasing anthropogenic load. Global climate change (Shimaraev and Domysheva, 2013) and anthropogenic impact on the Baikal ecosystem in recent years have contributed to the obvious changes in the structure of benthic communities from the coastal zone: abundant growth of filamentous algae, especially in places with high anthropogenic load; the mass development of cosmopolitan small-cell algae that are untypical for the lake and inhabit eutrophic water bodies; and, at the same time, a decrease in the number of endemic phytoplankton species (Kravtsova et al., 2012; 2014; Timoshkin et al., 2016; Bondarenko and Logacheva, 2017). In addition, since 2011 there has been a mass disease and mortality of sponges (Bormotov, 2012). Several types of disease in the Baikal sponges were described, all of which are characteristic of Lubomirskia baikalensis: bleaching, necrosis, violet cyanobacterial biofilm, and brown spots (Timoshkin et al., 2016; Khanaev et al., 2018). Notably, sponge disease is a well-known worldwide problem, and since 1983 the sponge disease outbreaks have been reported in a wide range of geographic locations, however, no diseases have been reported in freshwater sponge populations before (Webster, 2007). The understanding of the origin and causative agents of sponge disease is still insufficient. Some works indicated potential bacterial pathogens in sponges, however, most studies showed only shift in the microbial community of diseased sponges (dysbiosis) in comparison with healthy individuals (Webster, 2007; Belikov et al., 2018). To our knowledge, no viral putative pathogens have been found in diseased sponges.

The aim of this work was the study of the genetic diversity of DNA viruses in the associated community of the endemic Baikal sponge Lubomirskia baikalensis with signs of disease using metagenomic analysis. Viruses do not have universal genes, like the ribosomal genes of pro- and eukaryotes; therefore, the metagenomic analysis is still the most informative way to study the diversity of viral communities in natural samples. As far as we know, metagenomic studies of viruses in freshwater sponges have not been carried out previously.

\section{Materials and methods}

\section{Sampling, obtaining the viral fraction and DNA extraction}

The Lubomirskia baikalensis sponges were sampled using lightweight diving equipment in March 2015 in the Maloye More Strait of Lake Baikal, near the Malye Olkhonskiye Vorota Strait. Two branches of $2-3 \mathrm{~cm}^{3}$ in volume were collected from the same sponge. One branch looked healthy (sample Sv3h), and another had lesions in the form of brown spots (sample Sv3d) (Fig. 1). The sponge samples were washed twice in sterile water, frozen and transported to the laboratory. To obtain the fraction of virus-like particles, the samples were homogenized and centrifuged (3000 $\mathrm{rpm}, 30 \mathrm{~min}$ ); the aqueous fraction was passed through a syringe filter with a pore size of $0.2 \mu \mathrm{m}$ (Sartorius). Then, the samples were treated with DNase I and RNase A enzymes (Thermo Fisher Scientific) to remove contaminating nucleic acids. Viral DNA was extracted by the method with proteinase K, SDS and phenolchloroform extraction (Sambrook et al., 1989).

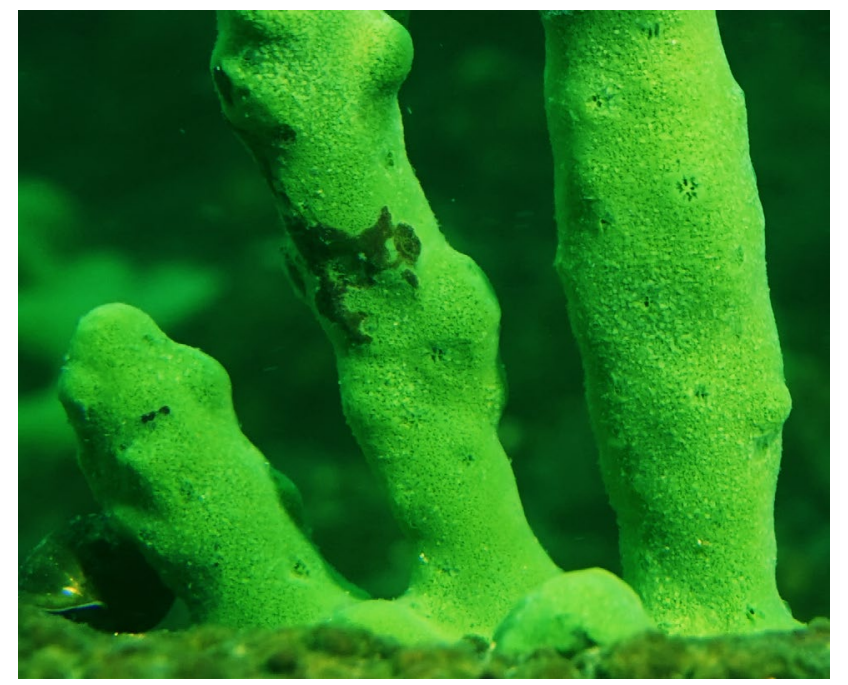

Fig. 1. Diseased sponge L. baikalensis with brown spots lesions 


\section{Library preparation and sequencing}

The total viral DNA was sheared in a microTUBE AFA Fiber Snap-cap using a Covaris S2 instrument (Covaris) with a medium size distribution of fragments of about 500 bp. The paired-end libraries were prepared using a NEBNext Ultra DNA library prep kit for Illumina (NEB). Sequencing of the virome was conducted on a MiSeq genome sequencer $(2 \times 300$ cycles, Illumina) in SB RAS Genomics Core Facility (ICBFM SB RAS, Novosibirsk, Russia).

\section{Analysis of virome datasets}

The primary processing of the received data (paired reads of $2 \times 300 \mathrm{bp}$ ) was performed using the R package "ShortReads" (Morgan et al., 2009). The sequences of less than 100 nucleotides were excluded before the next analysis.

Taxonomic identification of viral sequences was performed using the BLASTn algorithm (Altschul et al., 1990) against NCBI RefSeq complete viral genomes database (September 2018 release) (Pruitt et al., 2005). The BLASTn parameters used were as follows: cost to open a gap, two; cost to extend a gap, one; word size for word finder algorithm, twelve; penalty for a nucleotide mismatch, one; reward for a nucleotide match, one. The sequence reads were considered 'identified' if it had a relative in the reference database with an e-value of $<10^{-5}$ and bit score $\geq 50$. The results of BLASTn analysis were saved as a hit table. BLAST hits corresponding to the same viral genome subject ID were considered to belong to one virotype. Each subject ID from the BLASTn hit table was converted to a taxonomic annotation of the virus for a tabulated representation of the various virotypes in the sample. For further analysis, data on the representativeness of virotypes (number of reads per virotype in a sample) were normalized to the average number of reads per sample.

Rarefaction analysis was performed to assess the species richness in the samples (Heck et al., 1975). The representativeness of virotypes was used to calculate Shannon and Simpson indices of community biodiversity (Hill, 1973). Comparison of samples was carried out by cluster UPGMA method using the Bray-Curtis (Faith et al., 1987) and the Gower distances (Gower and Legendre, 1986). The clustering accuracy was estimated using the bootstrap method (1000 replicas). Before clustering, the representativeness of virotypes in samples was transformed into relative values (number of reads per virotype in a sample divided by total viral reads). The reliability of the difference between the distributions of virotypes representativeness in the compared communities was estimated using the chi square test. Statistical calculations were performed using the R packages "vegan" (Dixon, 2003) and "pvclust" (Suzuki and Shimodaira, 2006).

For a comparative analysis, other metaviromic datasets were used in this study: from the marine sponge Rhopaloeides odorabile (Great Barrier Reef (GBR), Davis Reef, sampled in January 2014; Laffy et al., 2016), from corals Acropora millepora (GBR, Orpheus Island Reef, sampled in March 2013) and Pocillopora damicornis
(GBR, Trunk Reef, November 2012; Weynberg et al., 2014). The reference data sets were also processed and analyzed according to the procedure described above.

\section{Results}

\section{viromes}

Identification of viral sequences in sponge

After processing and filtering of raw paired reads, 310080 high-quality sequences remained for the sample Sv3h and 327901 - for the sample Sv3d. The total number of sequences similar to the genomes of viruses in the RefSeq database for the samples of the sponge Lubomirskia baikalensis Sv3h and Sv3d was 6903 and 13432, respectively. This averaged to $2.23 \%$ and $4.1 \%$ of the total data sets, which is comparable with the analysis of reference metagenomic data sets (2.79\%, 3.35\% and $4.18 \%$ for samples of $P$. damicornis, $R$. odorabile and $A$. millepora, respectively).

The majority of the sequences in virome datasets from $L$. baikalensis were similar to double-stranded DNA (dsDNA) viruses, which is attributed to the method for preparing libraries for the MiSeq platform (Illumina), in which dsDNA has a significant advantage at the amplification stage (Kim and Bae, 2011). Thus, the proportion of single-stranded DNA (ssDNA) viruses did not exceed $0.8 \%$. We also detected a small number of RNA viruses $(0.16 \%$ and $0.54 \%$ in the Sv3h and Sv3d samples, respectively), among which there were not only reverse-transcribing viruses of the Retroviridae but also those from other families. We cannot explain the presence of reads similar to viral RNA, since we constructed and sequenced the libraries of total viral DNA. Further, we did not consider the sequences similar to RNA viruses.

\section{Taxonomic diversity of viral communities}

In this study, we have identified 259 and 293 virotypes in samples from the Baikal sponge Lubomirskia baikalensis (for the samples Sv3h and Sv3d, respectively), which belong to sixteen viral families (Fig. 2). The families Siphoviridae, Myoviridae, Podoviridae, Phycodnaviridae, Poxviridae, and Mimiviridae were the most numerous (represented by more than $1 \%$ of the sequences and accounted for approximately $97 \%$ of virome reads). The significant parts of viromes $(19.1 \%$ and $15.1 \%$ in the samples Sv3h and Sv3d, respectively) were unclassified to the family rank viruses (Fig. 2).

Bacteriophages (the families Siphoviridae, Myoviridae and Podoviridae) had the largest proportion in viromes of $L$. baikalensis, which was expectable considering the large abundance of bacteria in the sponge holobionts (up to $35 \%$ of the total sponge biomass at densities exceeding $10^{9}$ microbial cells per cubic centimetre of sponge tissue) (Hentschel et al., 2012). In the list of potential hosts for detected bacteriophages, the representatives of the phyla Proteobacteria prevailed (data not shown). As known, the members of the phyla Proteobacteria (especially, of the classes Alpha-, Gamma- and Deltaproteobacteria) are well-represented in the highly diverse sponge microbial 


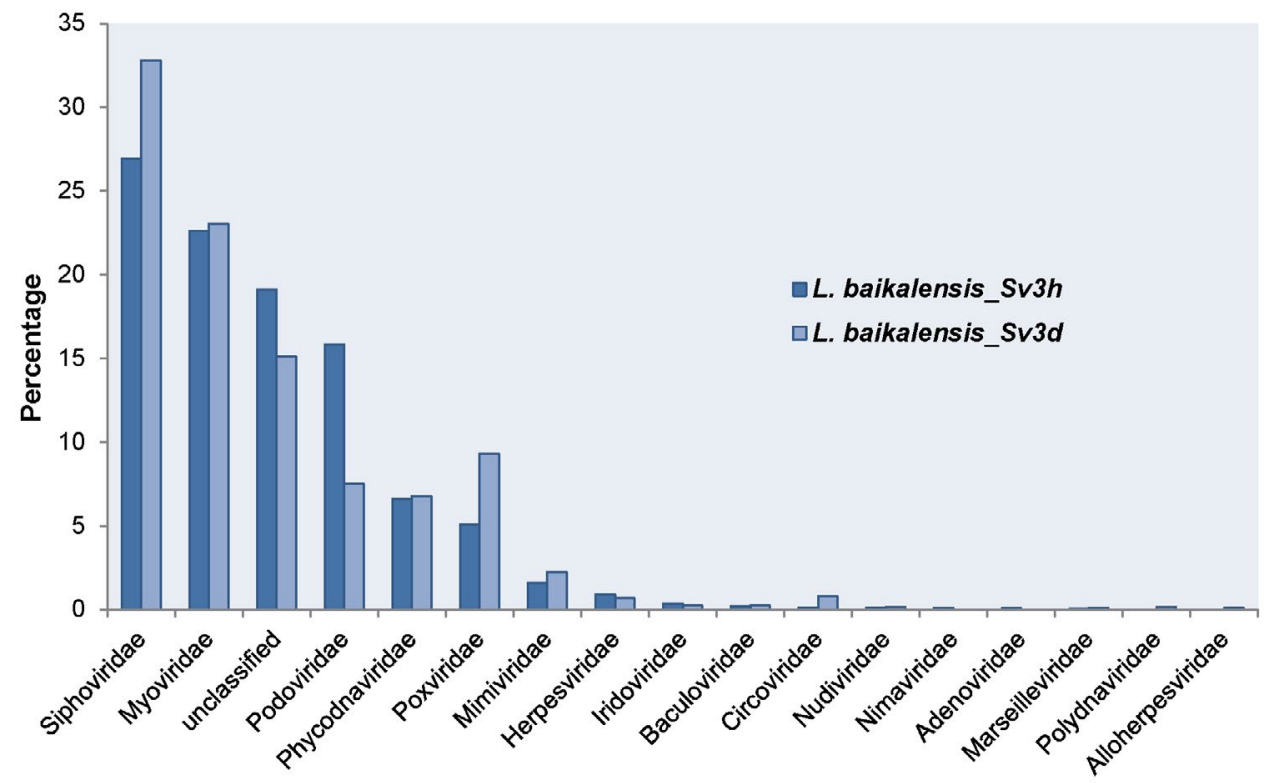

Fig. 2. Percentage of different viral families in viromes of Lubomirskia baikalensis

communities (Hentschel et al., 2012). In our study, the Alphaproteobacteria virus phiJl001, which infects a novel marine bacterium isolated from the marine sponge Ircinia strobilina (Lohr et al., 2005), has been detected among the identified virotypes. In addition, a great number of sequences were unexpectedly related to unclassified Idiomarinaceae phage 1N2-2. The Idiomarinaceae is a family within the order Alteromonadales of the class Gammaproteobacteria. Members of this family have been isolated from saline habitats, such as coastal and oceanic waters, solar salterns, submarine hydrothermal fluids and inland hypersaline wetlands (Albuquerque and da Costa, 2014). The Synechococcus phage S-CBP4 and Prochlorococcus phage P-SSM2 (Podoviridae and Myoviridae families, respectively) were also ones of the dominant (more than $1 \%$ of reads) putative bacteriophages in the Baikal sponge viromes.

Among the most abundant virotypes, there were also some eukaryotic viruses: viruses of microalgae Chrysochromulina ericina virus and Emiliania huxleyi virus 86 (the family Phycodnaviridae), the viruses of protozoa Yellowstone lake mimivirus, Megavirus chiliensis (Mimiviridae/Megaviridae) and Cedratvirus A11 (unclassified, putative Pithoviridae family), as well as BeAn 58058 virus (Poxviridae), and Yellowstone Lake virophage 5 (unclassified).

A comparative analysis of the diseased and the visually healthy sponge branches of $L$. baikalensis revealed that the composition of the most numerous families in two samples of the sponge was the same, but their percentage differed (Fig. 2). For example, the families Siphoviridae, Myoviridae, Phycodnaviridae, Poxviridae, and Mimiviridae were more abundant in the sample from the diseased branch of the sponge, and the families Podoviridae, Iridoviridae and Herpesviridae prevailed in the healthy one. Representatives of the families Nimaviridae and Adenoviridae were found only in the sample Sv3h, while Polydnaviridae and Alloherpesviridae were found only in Sv3d, but the number of reads for these families was low (less than 22). In general, the viromes of the visually healthy and diseased branches of $L$. baikalensis were significantly different ( $P$ value $<0.01$ ).

It should be noted that the number of reads was insufficient to estimate the total viral diversity in the samples of $L$. baikalensis, since the rarefaction curves in our analysis did not reach the plateau (data not shown). Thus, we estimated mainly only dominant spongeassociated viruses. In the future, it is necessary to carry out a deeper sequencing of virome libraries from the existing and/or new samples of the Baikal sponges.

\section{viromes}

Comparative analysis of sponge and coral

Figure 3 shows the proportions of viral families and unclassified at the rank of family viruses in the metagenomes of the Baikal sponge, marine sponge and corals. Tailed bacteriophages of the order Caudovirales (Myoviridae, Siphoviridae and Podoviridae families) dominated all datasets, but the distribution of these taxa differed between holobionts. Viral metagenome of Rhopaloeides odorabile was obvious for a greater number of the Myoviridae and vice versa - for a less number of the Siphoviridae in comparison with other metagenomes. The Podoviridae were the most numerous in the health branch of Lubomirskia baikalensis (Sv3h), as well as in corals Acropora millepora and Pocillopora damicornis. The families Phycodnaviridae, Poxviridae, Mimiviridae, and Herpesviridae also dominated all datasets, comprising $95-98 \%$ of reads together with tailed bacteriophages and unclassified viruses. The viromes of sponges differed from corals by a greater number of Phycodnaviridae and Mimiviridae. Unclassified viruses prevailed in $L$. baikalensis (Sv3h) and in $A$. millepora. The Herpesviridae were the most abundant in the sponge $R$. odorabile. The ssDNA viruses of the family Microviridae lacked in L. baikalensis while in the coral $P$. damicornis they accounted for $2.8 \%$. Variation between holobionts was also observed in composition and percentage of less numerous families (Fig. 3). The highest number of families (thirty) was detected in the marine sponge $R$. odorabile, which is most likely due to 
the largest number of reads.

UPGMA cluster analysis (considering the dominant virotypes, accounted for $95 \%$ of data sets) revealed two reliable clusters both, by using the BrayCurtis and the Gower distances. One cluster consisted of viral metagenomes from Baikal sponge, and other from the marine sponge and corals (Fig. 4).

\section{Discussion and conclusions}

In this study, we investigated viral communities of diseased sponge $L$. baikalensis through metagenomics. Despite the small number of reads, diversity indices of viruses in the $L$. baikalensis sponge were comparable to those in marine holobionts (Table 1), where the number of reads was much higher. Thus, we were able to identify a high diversity of viruses in samples of the Baikal sponge, and our analysis indicates the appropriateness of the chosen protocols for the isolation of VLPs and preparation of sponge viral DNA libraries for further studies of viral communities in the Baikal sponges.

Three bacteriophage families of the order Caudovirales: Siphoviridae, Myoviridae and Podoviridae mostly represented the L. baikalensis virome. This was expectable considering the high abundance and diversity of their bacterial hosts in associated communities. These viruses normally predominate in viromes of the aquatic biomes. Metagenomic studies of marine sponges also indicated the dominance of tailed bacteriophages (Laffy et al., 2016; 2018). The families Phycodnaviridae, Mimiviridae and Poxviridae were also numerous. Members of Phycodnaviridae infect microalgae; Mimiviridae are the largest viruses that infect amoeba and other protozoa. The dominance of viruses of these families in the sponge hologenome is also expectable considering the presence of their hosts in the communities of the Baikal sponges. At the same time, Claverie et al. (2009) show circumstantial

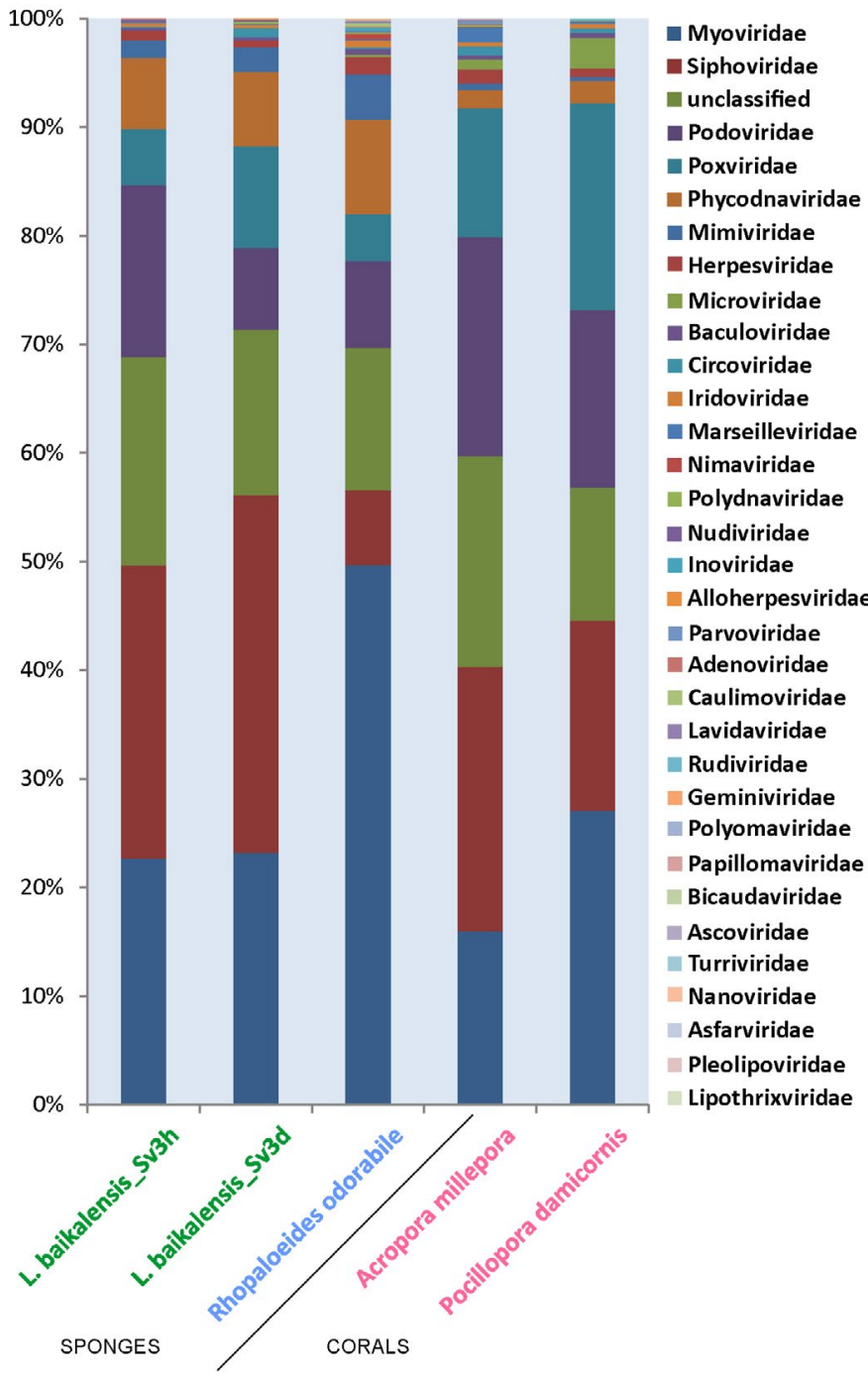

Fig. 3. The proportions of viral families and unclassified viruses in metaviromes of sponges and corals
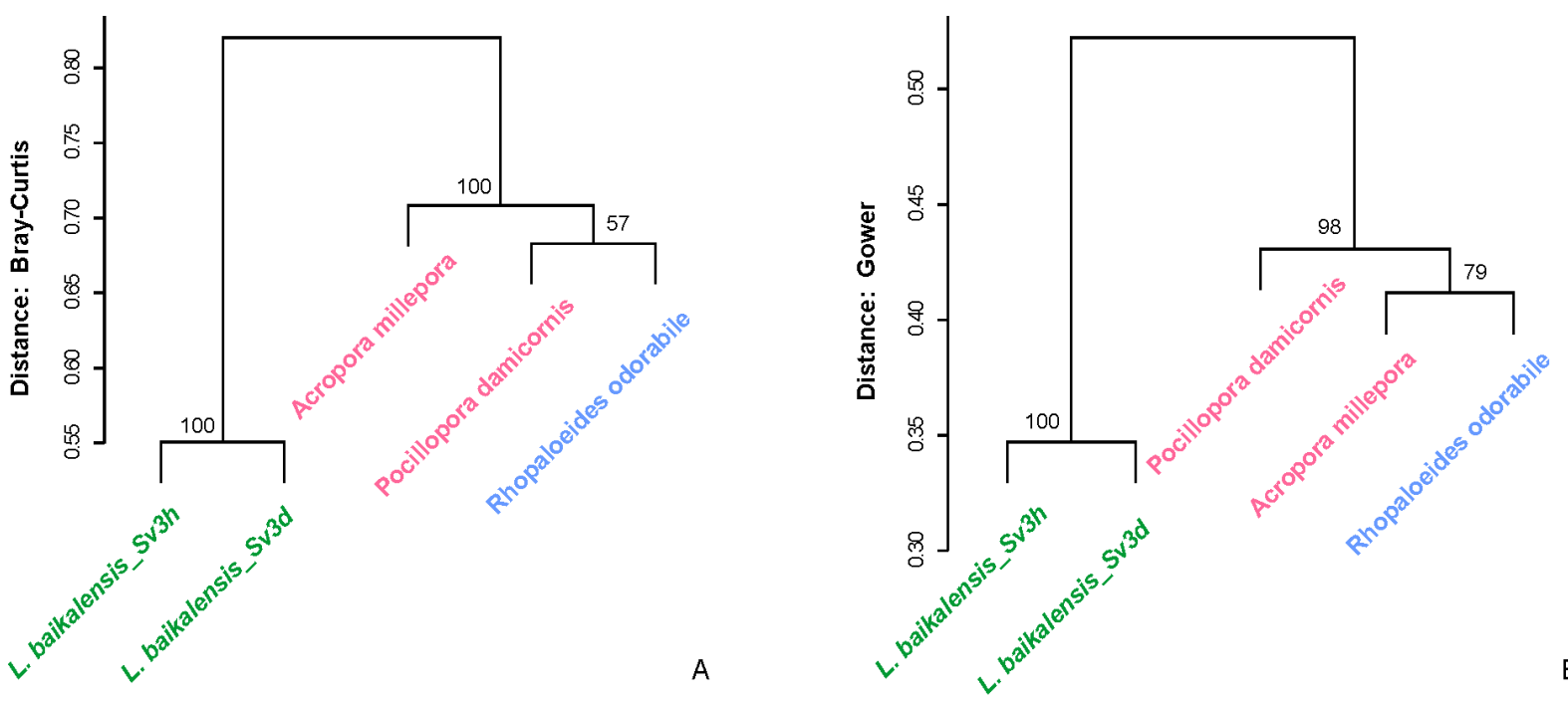

Fig. 4. UPGMA cluster analysis estimating the differences in diversity and composition of viromes performed using the BrayCurtis (A) and the Gower (B) distances. The samples from the Baikal sponge are marked in green, and those from marine sponge and corals - in blue and pink, respectively 
Table 1. Diversity indexes for the samples from sponges L. baikalensis and reference viromes

\begin{tabular}{|c|c|c|c|}
\hline Samples & Alpha diversity & Shannon index & Simpson index \\
\hline L. baikalensis_Sv3h & 259 & 4,699 & 0,981 \\
L. baikalensis_Sv3d & 293 & 4,348 & 0,965 \\
R. odorabile & 697 & 4,378 & 0,955 \\
A. millepora & 520 & 4,588 & 0,973 \\
P. damicornis & 813 & 5,028 & 0,959 \\
\hline
\end{tabular}

evidence of the possible mimivirus infection of some marine sponges (and corals).

It is worth noting that many viral families have a wide range of taxonomically distant hosts, and the list of species for the known viral families is constantly increasing. For example, known representatives of the heavily investigated family Poxviridae infect the widest host range among vertebrate and invertebrate taxa (Haller et al., 2014; Oliveira et al., 2017). Recently, new Salmon gill poxvirus (SGPV) has been found in the marine inhabitants (Gjessing et al., 2015). Like in our study, the sequences affiliated to Poxviridae were also detected in marine sponges Amphimedon queenslandica and Ianthella basta (Laffy et al., 2018). Therefore, it is most likely that the sequences of the sponge viromes similar to the known poxviruses of the terrestrial animals as well as other viruses of eukaryotes actually belong to unknown viruses that infect the aquatic invertebrates.

Despite the diversity of aquatic invertebrates, our knowledge of viral pathogens of this animal group including sponges is limited. Virus-like particles have been often identified without isolation of viruses and study of their pathogenesis in different species of invertebrates (Johnson, 1984; Munn, 2006). To date, several tens of aquatic invertebrates (mainly mollusks and crustaceans) viruses are known, and they are tentatively or more accurately assigned to the different families, including those found in the L. baikalensis sponge and other viromes: Herpesviridae, Baculoviridae, Iridoviridae, Adenoviridae, Nimaviridae, and Coronaviridae. In fact, previous studies have shown that herpes-like viruses are commonly observed in cnidarian viromes (Vega Thurber et al., 2008). It is suggested that herpes-like viruses infect all corals. Moreover, the sequences similar to Herpesviridae dominated samples of healthy corals but were less abundant in stressed or diseased coral tissues (Vega Thurber et al., 2008; Soffer et al., 2014). Thus, herpes-like viruses, as well as the abovementioned viruses of other families, may be a part of stable health sponge holobionts. The sponges provide food and habitat for many small invertebrates (mollusks, polychaetes, crustaceans and others) (Wulff, 2006), and those viruses could also be present in sponge viromes.

The diversity of a number of viral families belonging to the Nucleo-cytoplasmic large DNA viruses (NCLDVs) group was found in the sponge L. baikalensis and reference viromes, as well as in other studies of marine sponges (Laffy et al., 2016; 2018). NCLDVs is a monophyletic group of eukaryotic viruses with a large double-stranded DNA genome ranging from $100 \mathrm{~kb}$ to $1.26 \mathrm{Mb}$ (Yutin and Koonin, 2012). This group consists of seven families, five of which were found in the viromes L. baikalensis: Iridoviridae, Marseilleviridae, Mimiviridae, Phycodnaviridae, and Poxviridae. NCLDVs are known to have important roles in marine ecosystems (Hingamp et al., 2013) and most likely no less significant in sponges and other holobionts (Vega Thurber et al., 2008). Sequences similar to NCLDVs, including members of the Phycodnaviridae, Poxviridae and Mimiviridae were relatively more abundant in bleached coral tissues than in non-bleached ones (Soffer et al., 2014).

Mass disease of the Baikal sponges, throughout the entire water area of the lake, covering not only in the recreational but also in relatively favourable areas (Khanaev et al., 2018), indicates the infectious nature of the disease. However, the bacterial pathogens in the Baikal sponges have not been detected yet. As noted above, the viral pathogens of worldwide marine sponge diseases are also unknown and, unfortunately, the number of investigations in this field is the fewest. On the contrary, some viruses are known to be potential causative agents of coral diseases. It was suggested that eukaryotic circular Rep-encoding single-stranded DNA (CRESS-DNA) viruses (particularly Circoviridae and Nanoviridae), and their associated satellites may be responsible for white plague-like diseases of tropical corals (Soffer et al., 2014). Some sequences similar to circoviruses were also detected in the Baikal sponge viromes, but as mentioned above these ssDNA viruses were, probably, underestimated in our study. Therefore, the role of CRESS-DNA, as well as NCLDVs viruses in sponge holobionts, remains to be revealed in further examination of DNA-viruses (including ssDNA) in a number of diseased and healthy (without signs of disease) sponges.

Diseases of sponge and other holobionts are a result of stress due to the environmental perturbations and are accompanied by dysbiosis that is the substantial shifts in taxonomic and functional diversity of microbial communities (Pita et al., 2018). In this study, we analyzed the sponge with the signs of disease and, most likely, this individual also had dysbiosis and corresponding changes in abundances and composition of viral communities especially in the affected branch. This may explain a significant difference of viromes from the affected and visually healthy tissues of the $L$. baikalensis sponge. As a rule, the diversity of microbiota including viruses in diseased sponges is higher in 
comparison with the stable healthy holobiont (Pita et al., 2018), and in our study the number of virotypes in the diseased branch was a little more than in the visually healthy one.

All sponge- and coral-associated viral communities were significantly different in our analysis ( $P$ value $<0.01$ ); in addition, the freshwater viromes were distant from the marine ones (Fig. 4). This is consistent with the data reported previously, where the viruses associated with sponges were species specific (Laffy et al., 2018), and the environmental conditions affected the formation of viral communities (Batista et al., 2018). It should be noted that the methods of sample preparation influence on the results of metagenomic analysis; hence, some proportion of differences between the viromes from the Baikal and marine holobionts may be a consequence of this fact.

Therefore, the metagenomic analysis of the viral community from the Baikal sponges have revealed for the first time a high genetic and taxonomic diversity of viruses in the associated communities of the L. baikalensis sponges, and demonstrated the differences in viral communities of visually healthy and diseased branches of the sponge. In general, our study broadens the understanding of the nature of symbiotic communities in freshwater organisms. Future investigations are necessary to assess the diversity and functions of viral communities in different species of the Baikal sponges; understand the role of viruses in mass disease and mortality of sponges; and trace the dynamics of viral communities in the course of the disease until adaptation of sponge holobionts and the onset of a new stable equilibrium state.

\section{Acknowledgments}

The study was carried out within the framework of the state task No. 0345-2019-0002 and supported by the funding of RFBR and the Government of the Irkutsk Region, project No. 17-44-388080.

\section{References}

Albuquerque L., da Costa M.S. 2014. The family Idiomarinaceae. In: Rosenberg E., DeLong E.F., Lory S. et al. (Eds.), The Prokaryotes. Heidelberg, pp. 361-385. DOI: 10.1007/978-3-642-38922-1_232

Altschul S.F., Gish W., Miller W. et al. 1990. Basic local alignment search tool. Journal of Molecular Biology 215: 403-410. DOI: 10.1016/S0022-2836(05)80360-2

Batista D., Costa R., Carvalho A.P. et al. 2018. Environmental conditions affect activity and associated microorganisms of marine sponges. Marine Environmental Research 142: 59-68. DOI: 10.1016/j.marenvres.2018.09.020

Belikov S.I., Feranchuk S.I., Butina T.V. et al. 2018. Mass disease and mortality of Baikal sponges. Limnology and Freshwater Biology 1: 36-42. DOI: 10.31951/2658-3518-2018-A-1-36

Bell J.J. 2008. The functional roles of marine sponges. Estuarine, Coastal and Shelf Science 79: 341-353. DOI: 10.1016/j.ecss.2008.05.002

Bondarenko N.A., Logacheva N.F. 2017. Structural changes in phytoplankton of the littoral zone of Lake Baikal. Hydrobiological Journal 53: 16-24. DOI: 10.1615/HydrobJ. v53.i2.20

Bormotov A.E. 2012. What has happened to Baikal sponges? Science First Hand 32: 20-23.

Butina T.V., Potapov S.A., Belykh O.I. et al. 2015. Genetic diversity of cyanophages of the myoviridae family as a constituent of the associated community of the Baikal sponge Lubomirskia baicalensis. Russian Journal of Genetics 51: 313-317. DOI: 10.1134/S1022795415030011

Claverie J.M., Grzela R., Lartigue A. et al. 2009. Mimivirus and Mimiviridae: giant viruses with an increasing number of potential hosts, including corals and sponges. Journal of Invertebrate Pathology 101: 172-180. DOI: 10.1016/j. jip.2009.03.011

Diaz M.C., Rützler K., 2001. Sponges: an essential component of Caribbean coral reefs. Bulletin of Marine Science 69: 535-546.

Dixon P. 2003. VEGAN, a package of $\mathrm{R}$ functions for community ecology. Journal of Vegetation Science 14: 927-930. DOI:10.1658/1100-9233(2003)014[0927:vaporf]2 $.0 . c 0 ; 2$

Efremova S.M. 2001. Sponges (Porifera). In: Timoshkin O.A. (Ed.), Index of animal species inhabiting Lake Baikal and its catchment area. Novosibirsk, pp. 182-192. (in Russian)

Efremova S.M. 2004. New genus and new species of sponges from family Lubomirskiidae Rezvoj, 1936. In: Timoshkin O.A. (Ed.), Index of animal species inhabiting Lake Baikal and its catchment area. Novosibirsk, pp. 1261-1278. (in Russian)

Faith D.P., Minchin P.R., Belbin L. 1987. Compositional dissimilarity as a robust measure of ecological distance. Vegetatio 69: 57-68. DOI: 10.1007/BF00038687

Gjessing M.C., Yutin N., Tengs T. et al. 2015. Salmon gill poxvirus, the deepest representative of the Chordopoxvirinae. Journal of Virology 89: 9348-9367. DOI: 10.1128/ JVI.01174-15

Gower J.C., Legendre P. 1986. Metric and Euclidean properties of dissimilarity coefficients. Journal of Classification 3: 5-48. DOI: 10.1007/BF01896809

Haller S.L., Peng C., McFadden G. et al. 2014. Poxviruses and the evolution of host range and virulence. Infection, Genetics and Evolution 21: 15-40. DOI: 10.1016/j. meegid.2013.10.014

Heck Jr. K.L., van Belle G., Simberloff D. 1975. Explicit calculation of the rarefaction diversity measurement and the determination of sufficient sample size. Ecology 56: 14591461. DOI: $10.2307 / 1934716$

Hentschel U., Piel J., Degnan S.M. et al. 2012. Genomic insights into the marine sponge microbiome. Nature Reviews Microbiology 10: 641-654. DOI: 10.1038/nrmicro2839

Hill M.O. 1973. Diversity and evenness: a unifying notation and its consequences. Ecology 54: 427-432. DOI: $10.2307 / 1934352$

Hingamp P., Grimsley N., Acinas S.G. et al. 2013. Exploring nucleo-cytoplasmic large DNA viruses in Tara Oceans microbial metagenomes. The ISME Journal 7: 16781695. DOI: $10.1038 /$ ismej.2013.59

Itskovich V., Kaluzhnaya O., Veynberg Y. et al. 2017. Endemic Lake Baikal sponges from deep water. 2: Study of the taxonomy and distribution of deep-water sponges of Lake Baikal. Zootaxa 4236: 335-342. DOI: 10.11646/ zootaxa.4236.2.8

Jacquet S., Miki T., Noble R. et al. 2010. Viruses in aquatic ecosystems: important advancements of the last 20 years and prospects for the future in the field of microbial oceanography and limnology. Advances in Oceanography and Limnology 1: 97-141. DOI: 10.1080/19475721003743843

Johnson P.T. 1984. Viral diseases of marine invertebrates. Helgoländer Meeresuntersuchungen [Heligoland Marine Surveys] 37: 65-98. 
Khanaev I.V., Kravtsova L.S., Maikova O.O. et al. 2018. Current state of the sponge fauna (Porifera: Lubomirskiidae) of Lake Baikal: sponge disease and the problem of conservation of diversity. Journal of Great Lakes Research 44: 77-85. DOI:10.1016/j.jglr.2017.10.004

Kim K.H., Bae J.W. 2011. Amplification methods bias metagenomic libraries of uncultured single-stranded and double-stranded DNA viruses. Applied and Environmental Microbiology 77: 7663-7668. DOI: 10.1128/AEM.00289-11

Kozhov M.M. 1970. About the benthos of south Baikal. Izvestiya BGNII pri IGU [Bulletin of the Biological and Geographical Research Institute at the Irkutsk State University] 23: 3-12. (in Russian)

Kozhova O.M., Izmesteva L.R. 1998. Lake Baikal: Evolution and biodiversity. Leiden: Backhuys Publisher.

Kravtsova L.S., Izhboldina L.A., Khanaev I.V. et al. 2012. Disturbances of the vertical zoning of green algae in the coastal part of the Listvennichnyi Gulf of Lake Baikal. Doklady Biological Sciences 448: 227-229. DOI: 10.1134/ S0012496612060026

Kravtsova L.S., Izhboldina L.A., Khanaev I.V. et al. 2014. Nearshore benthic blooms of filamentous green algae in Lake Baikal. Journal of Great Lakes Research 40: 441-448. DOI: 10.1016/j.jglr.2014.02.019

Laffy P.W., Wood-Charlson E.M., Turaev D. et al. 2016. HoloVir: a workflow for investigating the diversity and function of viruses in invertebrate holobionts. Frontiers in Microbiology 7: 822. DOI: 10.3389/fmicb.2016.00822

Laffy P.W., Wood-Charlson E.M., Turaev D. et al. 2018. Reef invertebrate viromics: diversity, host specificity and functional capacity. Environmental Microbiology 20: 21252141. DOI: $10.1111 / 1462-2920.14110$

Lohr J.E., Chen F., Hill R.T. 2005. Genomic analysis of bacteriophage ФJL001: insights into its interaction with a sponge-associated alpha-proteobacterium. Applied and Environmental Microbiology 71: 1598-1609. DOI: 10.1128/ AEM.71.3.1598-1609.2005

Morgan M., Anders S., Lawrence M. et al. 2009. ShortRead: a bioconductor package for input, quality assessment and exploration of high-throughput sequence data. Bioinformatics 25: 2607-2608. DOI: 10.1093/bioinformatics/btp450

Munn C.B. 2006. Viruses as pathogens of marine organisms - from bacteria to whales. Journal of the Marine Biological Association of the United Kingdom 86: 453-467. DOI: $10.1017 /$ S002531540601335X

Oliveira G.P., Rodrigues R., Lima M.T. et al. 2017. Poxvirus host range genes and virus-host spectrum: a critical review. Viruses 9: 331. DOI:10.3390/v9110331

Pascelli C., Laffy P.W., Kupresanin M. et al. 2018. Morphological characterization of virus-like particles in coral reef sponges. PeerJ 6. DOI:10.7717/peerj.5625

Pita L., Rix L., Slaby B.M. et al. 2018. The sponge holobiont in a changing ocean: from microbes to ecosystems. Microbiome 6: 46. DOI:10.1186/s40168-018-0428-1

Rohwer F., Seguritan V., Azam F. et al. 2002. Diversity and distribution of coral-associated bacteria. Marine Ecology Progress Series 243: 1-10. DOI: 10.3354/meps243001

Rosenberg E., Koren O., Reshef L. et al. 2007. The role of microorganisms in coral health, disease and evolution. Nature Reviews Microbiology 5: 355-362. DOI: 10.1038/ nrmicro1635

Pruitt K.D., Tatusova T., Maglott D.R. 2005. NCBI
Reference Sequence (RefSeq): a curated non-redundant sequence database of genomes, transcripts and proteins. Nucleic Acids Research 33. DOI: 10.1093/nar/gki025

Sambrook J., Fritsch E.F., Maniatis T. 1989. Molecular cloning: a laboratory manual (Ed. 2). New York: Cold spring harbor laboratory press.

Shimaraev M.N., Domysheva V.M. 2013. Trends in hydrological and hydrochemical processes in Lake Baikal under conditions of modern climate change. In: Goldman C.R., Kumagai M., Robarts R.D. (Eds.), Climatic change and global warming of inland waters. Impacts and mitigation for ecosystems and societies. Chichester, pp. 43-66. DOI 10.1002/9781118470596.ch3

Soffer N., Brandt M.E., Correa A.M. et al. 2014. Potential role of viruses in white plague coral disease. The ISME Journal 8: 271-283. DOI: 10.1038/ismej.2013.137

Suttle C.A. 2007. Marine viruses - major players in the global ecosystem. Nature Reviews Microbiology 5: 801-812. DOI: $10.1038 /$ nrmicro1750

Suzuki R., Shimodaira H. 2006. Pvclust: an R package for assessing the uncertainty in hierarchical clustering. Bioinformatics 22: 1540-1542. DOI: 10.1093/bioinformatics/ btl117

Timoshkin O.A., Samsonov D.P., Yamamuro M. et al. 2016. Rapid ecological change in the coastal zone of Lake Baikal (East Siberia): is the site of the world's greatest freshwater biodiversity in danger? Journal of Great Lakes Research 42: 487-497. DOI:10.1016/j.jglr.2016.02.011

Vacelet J., Gallissian M.F. 1978. Virus-like particles in cells of the sponge Verongia cavernicola (Demospongiae, Dictyoceratida) and accompanying tissues changes. Journal of Invertebrate Pathology 31: 246-254. DOI 10.1016/0022-2011(78)90014-9

Vega Thurber R.L., Barott K.L., Hall D. et al. 2008. Metagenomic analysis indicates that stressors induce production of herpes-like viruses in the coral Porites compressa. Proceedings of the National Academy of Sciences of the United States of America 105: 18413-18418. DOI: 10.1073/ pnas.0808985105

Webster N.S. 2007. Sponge disease: a global threat? Environmental Microbiology 9: 1363-1375. DOI: 10.1111/j.1462-2920.2007.01303.x

Webster N.S., Thomas T. 2016. The Sponge Hologenome. mBio 7. DOI:10.1128/mBio.00135-16

Weynberg K.D., Wood-Charlson E.M., Suttle C.A. et al. 2014. Generating viral metagenomes from the coral holobiont. Frontiers in Microbiology 5: 206. DOI: 10.3389/ fmicb.2014.00206

Wilhelm S.W., Matteson A.R. 2008. Freshwater and marine virioplankton: a brief overview of commonalities and differences. Freshwater Biology 53: 1076-1089. DOI: 10.1111/j.1365-2427.2008.01980.x

Wommack K.E., Colwell R.R. 2000. Virioplankton: viruses in aquatic ecosystems. Microbiology and Molecular Biology Reviews 64: 69-114.

Wulff J.L. 2006. Ecological interactions of marine sponges. Canadian Journal of Zoology 84: 146-166. DOI:10.1139/ Z06-019

Yutin N., Koonin E.V. 2012. Hidden evolutionary complexity of nucleo-cytoplasmic large DNA viruses of eukaryotes. Virology Journal 9: 161. DOI: $10.1186 / 1743-422 X-9-161$ 